\title{
Direct numerical simulation tests of eddy viscosity in two dimensions
}

\author{
A. Chekhlov and S.A. Orszag \\ Program in Applied and Computational Mathematics, Princeton University, \\ Princeton, NJ 08544 \\ S. Sukoriansky \\ Department of Mechanical Engineering, Ben-Gurion University of the Negev, \\ Beer-Sheva 84105, Israel \\ B. Galperin \\ Department of Marine Science, University of South Florida, \\ St. Petersburg, FL 33701 \\ I. Staroselsky \\ Cambridge Hydrodynamics, Inc., P.O. Box 1403, Princeton, NJ 08542
}

(November 21, 2018)

\begin{abstract}
Two-parametric eddy viscosity (TPEV) and other spectral characteristics of two-dimensional (2D) turbulence in the energy transfer sub-range are calculated from direct numerical simulation (DNS) with $512^{2}$ resolution. The DNS-based TPEV is compared with those calculated from the test field model (TFM) and from the renormalization group (RG) theory. Very good agree-
\end{abstract}


ment between all three results is observed.

Typeset using REVTEX 
Two-dimensional incompressible turbulent flows are described by the vorticity equation:

$$
\frac{\partial \zeta}{\partial t}+\frac{\partial\left(\nabla^{-2} \zeta, \zeta\right)}{\partial(x, y)}=\nu_{0} \nabla^{2} \zeta
$$

where $\zeta$ is fluid vorticity and $\nu_{0}$ is molecular viscosity. It is well known that the existence of inviscid invariants $\int d^{2} x \zeta^{2 n}$ of (II) results in the flux of energy towards the largest spatial scales. The presence of this inverse cascade complicates the large-scale description of 2D flows and requires refinement of the classical hydrodynamic notion of "eddy viscosity." The concept of eddy viscosity is well defined for 3D turbulent flows, where energy cascades towards the smallest flow scales where it is dissipated. To achieve an adequate coarsegrained description of 3D flow, one can introduce increased "effective" dissipation at large scales which accounts for the unresolved dissipation.

In 2D flows, the inverse flux of energy at large scales and enstrophy dissipation at small scales make the eddy viscosity concept more subtle. It was argued by Kraichnan [1] that, in Fourier space, a 2D eddy viscosity should include two parameters: a cutoff wave number $k_{c}$ (which essentially determines the size of the coarse grain), and the wave number of a given mode, $k$. The two-parameter eddy viscosity (TPEV), denoted by $\nu\left(k \mid k_{c}\right)$, describes the energy exchange between a given resolved vorticity mode with the wave number $k$ and all subgrid, or unresolved, modes with $k>k_{c}$; it provides correct account for the energy and enstrophy fluxes between resolved and unresolved scales. The TPEV is derived from the evolution equation for the spectral enstrophy density $\Omega(k, t) \equiv \pi k\langle\zeta(\mathbf{k}, t) \zeta(-\mathbf{k}, t)\rangle$, where $\langle\ldots\rangle$ denotes averaging over thin circular shells:

$$
\left(\partial_{t}+2 \nu k^{2}\right) \Omega(k, t)=T_{\Omega}(k, t)
$$

Here, the enstrophy transfer function $T_{\Omega}(k, t)$ is given by

$$
T_{\Omega}(k, t)=\pi k \int_{\mathbf{p}+\mathbf{q}=\mathbf{k}} \frac{\mathbf{p} \times \mathbf{q}}{p^{2}}\langle\zeta(\mathbf{p}, t) \zeta(\mathbf{q}, t) \zeta(-\mathbf{k}, t)\rangle \frac{d \mathbf{p} d \mathbf{q}}{(2 \pi)^{2}}+\text { c.c. },
$$

where c.c. stands for the complex conjugate term. Assuming that the system is in statistical steady state and extending integration in (3) only over all such triangles (k, p, q) that 
$|k-p|<q<k+p$ and $p$ and/or $q$ are greater than $k_{c}$, one defines the two-parametric transfer $T_{\Omega}\left(k \mid k_{c}\right)$ and TPEV [1]:

$$
\nu\left(k \mid k_{c}\right)=-\frac{T_{\Omega}\left(k \mid k_{c}\right)}{2 k^{2} \Omega(k)}
$$

In a wide class of quasi-normal approximations [2] the two-parametric transfer $T_{\Omega}\left(k \mid k_{c}\right)$ in two dimensions is given by

$$
\begin{aligned}
T_{\Omega}\left(k \mid k_{c}\right) & =\iint_{\Delta} \Theta_{-k, p, q}\left(p^{2}-q^{2}\right) \sin \alpha\left[\frac{p^{2}-q^{2}}{p^{2} q^{2}} \Omega(p) \Omega(q)\right. \\
& \left.-\frac{k^{2}-q^{2}}{k^{2} q^{2}} \Omega(q) \Omega(k)+\frac{k^{2}-p^{2}}{k^{2} p^{2}} \Omega(p) \Omega(k)\right] d p d q
\end{aligned}
$$

where $\Theta_{-k, p, q}$ is the triad relaxation time. Here, the angle $\alpha$ is formed by the vectors $\mathbf{p}$ and $\mathbf{q}$, and $\iint_{\Delta}$ denotes integration over the area defined above (4).

The main difference between various spectral closure models is in specification of $\Theta_{-k, p, q}$. In [1],$T_{\Omega}\left(k \mid k_{c}\right)$ was evaluated using TFM. It was found that TPEV is a sign-changing function of the form $\nu\left(k \mid k_{c}\right)=\left|\nu\left(0 \mid k_{c}\right)\right| N\left(k / k_{c}\right)$, with $\nu\left(0 \mid k_{c}\right)<0, N(0)=-1$, and $N(1) \approx$ 2.1. The derivation of $\Theta_{-k, p, q}$ using the RG theory was given in [3] and adapted for 2D isotropic and anisotropic turbulence in [4] and [5], respectively. In the present work, we compare $\nu\left(k \mid k_{c}\right)$ for the inverse energy cascade regime calculated from DNS data with those predicted by TFM and the RG theory.

We solve Eq. (四) numerically in a periodic box of the size $2 \pi \times 2 \pi$ using $512^{2}$ Fourier modes. The numerical scheme involves a Fourier-Galerkin pseudo-spectral spatial approximation with implicit Adams-type second order stiffly stable time-stepping scheme [6]. In order to increase the effective inertial range, mode-selective hyperviscosity [7] of the form $\nu(k)=\nu_{L}(k)+\nu_{S}(k)=A_{L} k^{-10}+A_{S} k^{14}$ has been introduced in the vorticity equation (1) instead of the molecular viscosity. The constant coefficients $A_{L}$ and $A_{S}$ have been selected empirically so as to minimize distortion of the energy inertial subrange.

To simulate the inverse energy cascade, high wave number forcing

$$
f(k, t)=A_{f}\left(\delta_{k, k_{f}-1}+\delta_{k, k_{f}}+\delta_{k, k_{f}+1}\right) e^{i \phi(t)}
$$


is introduced in the vorticity equation; here, $A_{f}$ is the forcing amplitude, and random variable $\phi(t)$ is a white-noise function of time $t$ uniformly distributed in the interval $[0,2 \pi]$. The results are not sensitive to initial vorticity field distributions, including the extreme case of the zero field. A series of numerical experiments with various $k_{f}$ and other flow parameters have been performed. Here we shall report only the results with $k_{f}=98$ since they gave the broadest inertial range. Other parameters used in these simulations were $A_{f}=0.03244, A_{L}=0.5$, and $A_{S}=0.95 \times 10^{-34}$. The value of $A_{S}$ chosen is somewhat high, to ensure efficient enstrophy dissipation and to eliminate the need for the dealiasing.

In Figs 1a,b we plot the total energy $E_{\text {tot }}(t)=\int_{0}^{+\infty} k^{-2} \Omega(k, t) d k$ and enstrophy $\Omega_{\text {tot }}(t)=$ $\int_{0}^{+\infty} \Omega(k, t) d k$ as functions of time. In Fig. 1a, one can see that the energy grows with time and eventually tends to reach a steady state. However, the drift towards the energy steady state is significantly slower than towards that of the enstrophy. Defining the rms velocity as $V_{\mathrm{rms}}^{2}=\sum_{\mathbf{k}}|\mathbf{u}(\mathbf{k})|^{2}$ and the characteristic turnover time of the largest eddies as $\tau_{t u}=2 \pi / V_{\mathrm{rms}}$, we infer from Fig. 1b that a steady state for the total enstrophy was achieved after about $1.2 \tau_{t u}$, while about $5 \tau_{t u}$ were required to attain a steady state for the total energy. Note however that all the modes with $k>5$ have reached the steady state after $t \approx 2 \tau_{t u}$, and only the largest modes were still developing. The results presented below pertain to the integration time $t \leq 10^{4}$, before the energy saturates at low wave numbers.

In Fig. 2 we plot the time-averaged energy spectrum obtained after about $5 \tau_{t u}$. The inertial range $E \propto k^{-x}$ extends over more than a decade in wave number space. Mean square line-fitting over the interval $k \in(12,50)$ gives the scaling exponent close to the Kolmogorov value of $\frac{5}{3}$. Note that good agreement with the Kolmogorov scaling in the energy sub-range has been reported recently in [8] for $256^{2}$ simulations and in [9] for very high resolution simulations with $2048^{2}$ Fourier modes. In Fig. 2 we also plot a compensated energy spectrum, $k^{5 / 3} \epsilon^{-2 / 3} E(k)$, where $\epsilon$ is the energy transfer rate. The value of the Kolmogorov constant calculated from this data is about $C_{k}=6.2$, in reasonable agreement with 5.8 , calculated from DNS in [10] using the $256^{2}$ resolution and 6.69, obtained analytically in [11] on the basis of TFM. 
In Figs 3a,b we plot the $k$-dependent energy and enstrophy flux functions, defined as $\Pi_{E}(k)=\int_{0}^{k} T_{\Omega}(n) n^{-2} d n$ and $\Pi_{\Omega}(k)=\int_{0}^{k} T_{\Omega}(n) d n$, respectively. As expected, an inverse energy cascade with constant energy transfer rate $\epsilon$ develops for $k<k_{f}=98$, see Fig. 3a. For $k>k_{f}, \Pi_{E}(k)$ and $\Pi_{\Omega}(k)$ both quickly fall to zero, due to the strong dissipation at wave numbers adjacent to $k_{f}$. In Fig. 3b, we see that the flux of enstrophy in the energy sub-range, $k<k_{f}$, is zero. Strong enstrophy flux is observed for $k>k_{f}$, until the enstrophy dissipation takes over and suppresses the flux of enstrophy into even smaller scales. The resolution employed in this study was insufficient to detect a well defined enstrophy transfer range. The results plotted in Figs 3a,b also indicate that the numerical scheme used conserves both total energy and enstrophy, since $\Pi_{\Omega}(0)=\Pi_{\Omega}(\infty)=0$ and $\Pi_{E}(0)=\Pi_{E}(\infty)=0$.

We have calculated $k$ and $k_{c}$-dependent enstrophy transfer function $T_{\Omega}\left(k \mid k_{c}\right)$ employed in (4) by computing the third-order vorticity cumulants in (3) extending the integration only over those $\mathbf{p}$ and $\mathbf{q}$ that either $p \geq k_{c}$ or $q \geq k_{c}$. We have set $k_{c}=50$, well inside the energy inertial subrange.

The DNS-inferred normalized TPEV [ viz., the function $N\left(k / k_{c}\right)=\nu\left(k \mid k_{c}\right) /\left|\nu\left(0 \mid k_{c}\right)\right|$ ] is plotted in Fig. 4, along with the TFM-based [1] and RG-based [4] analytical predictions. The agreement between the DNS-based results and the TFM and RG theories is very good over the entire energy transfer range, up to the wave numbers close to $k_{c}$, where the DNS data saturates, while TFM and RG curves exhibit sharp cusp. The physics leading to this cusp is as follows. As closer $k$ approaches $k_{c}$, as more elongated triads with either $p$ or $q \ll k_{c}$ become involved in the energy exchange between the mode $k$ and the subgrid scale modes. The contribution from these triads results in the cusp behavior of the theoretical TPEV. However, in finite box DNS with large-scale energy removal, the energy of small wave number modes is reduced (see Fig. 2), which implies that instead of the sharp growth, the TPEV should saturate at $k \rightarrow k_{c}$. To illustrate and quantify this explanation, we recalculated the RG-based TPEV with the enstrophy spectrum in (5) corrected at $k \leq 5$ according to Fig. 2. In Fig. 5, we compare the DNS- and RG-based TPEV in their actual values, whereas the RG calculations were based upon the value of $\epsilon$ found from DNS. The agreement between 
the two is very good. We have also calculated TPEV for $k_{c}=35,45$ and 55 and found that the DNS-inferred TPEV scales with $k_{c}^{-4 / 3}$, in full agreement with the Kolmogorov and Richardson laws. At all values of $k_{c}$ tested an equally good agreement between DNS data and RG predictions was observed.

The good agreement demonstrated in Figs. 4 and 5 provides an indirect validation of TFM and RG results for isotropic 2D turbulence.

\section{ACKNOWLEDGMENTS}

Authors would like to thank Eric Jackson for valuable help with some programming issues, and Robert Kraichnan who kindly provided his data for TFM-based eddy viscosity. This research has been partially supported by ONR Grants N00014-92-J-1363, N00014-92C-0089 and N00014-92-C-0118, NSF Grant OCE 9010851, and the Perlstone Center for the Aeronautical Engineering Studies. The computations were performed on Cray Y-MP of NAVOCEANO Supercomputer Center, Stennis Space Center, Mississippi. 


\section{REFERENCES}

[1] R. H. Kraichnan, "Eddy viscosity in two and three dimensions," J. Atmos. Sci. 33, $1521(1976)$.

[2] W. D. McComb, The Physics of Fluid Turbulence (Oxford Science Publications, 1991).

[3] W. P. Dannevik, V. Yakhot and S. A. Orszag, "Analytical theories of turbulence and the $\epsilon$ expansion," Phys. Fluids 30, 2021 (1987).

[4] I. Staroselsky and S. Sukoriansky, "Renormalization group approach to two-dimensional turbulence and the $\epsilon$-expansion for the vorticity equation," in: Advances in Turbulence Studies 149, 159 (Progress in Astron. and Aeron., eds. H. Branover and Y. Unger, AIAA, 1993).

[5] B. Galperin, S. Sukoriansky and I. Staroselsky, "Eddy Rossby wave frequency in $\beta$-plane turbulence," Phys. Fluids A 5, in press (1993).

[6] G. E. Karniadakis, M. Israeli, S. A. Orszag, "High-order splitting methods for the incompressible Navier-Stokes equations," J. Comp. Phys. 97, 414 (1991).

[7] B. Legras, P. Santangelo and R. Benzi, "High-resolution numerical experiments for forced two-dimensional turbulence," Europhys. Lett. 5, 37 (1988).

[8] M. E. Maltrud and G. K. Vallis, "Energy and enstrophy transfer in numerical simulations of two-dimensional turbulence," Phys. Fluids A 5, 1760 (1993).

[9] L. M. Smith and V. Yakhot, "Bose condensation and small-scale structure generation in a random force driven 2D turbulence," Phys. Rev. Lett 71, 352 (1993).

[10] M. E. Maltrud and G. K. Vallis, "Energy spectra and coherent structures in forced two-dimensional and beta-plane turbulence," J. Fluid Mech. 228, 321 (1991).

[11] R. H. Kraichnan, "Inertial-range transfer in two- and three-dimensional turbulence," J. Fluid Mech. 3, 47 (1971). 


\section{FIGURES}

FIG. 1. Evolution of the total energy $E(k)$ (dotted line) and enstrophy $\Omega(k)$ (solid line) towards the steady state. Dashed line denotes the total energy with the first 6 modes excluded.

FIG. 2. Energy spectrum $E(k)$ (solid line) and compensated energy spectrum $E(k) k^{5 / 3} \epsilon^{-2 / 3}$ (dotted line).

FIG. 3. The energy flux $\Pi_{E}(k)$ (solid line) and the enstrophy flux $\Pi_{\Omega}(k)$ (dotted line).

FIG. 4. Normalized two-parametric eddy viscosity from DNS (dots), from TFM (dashed line), and from RG (solid line).

FIG. 5. Actual two-parametric eddy viscosity from DNS (dots) and from RG (solid line). In RG calculations, the energy spectrum for $k<5$ was corrected in accordance with the DNS results, Fig. 2. 


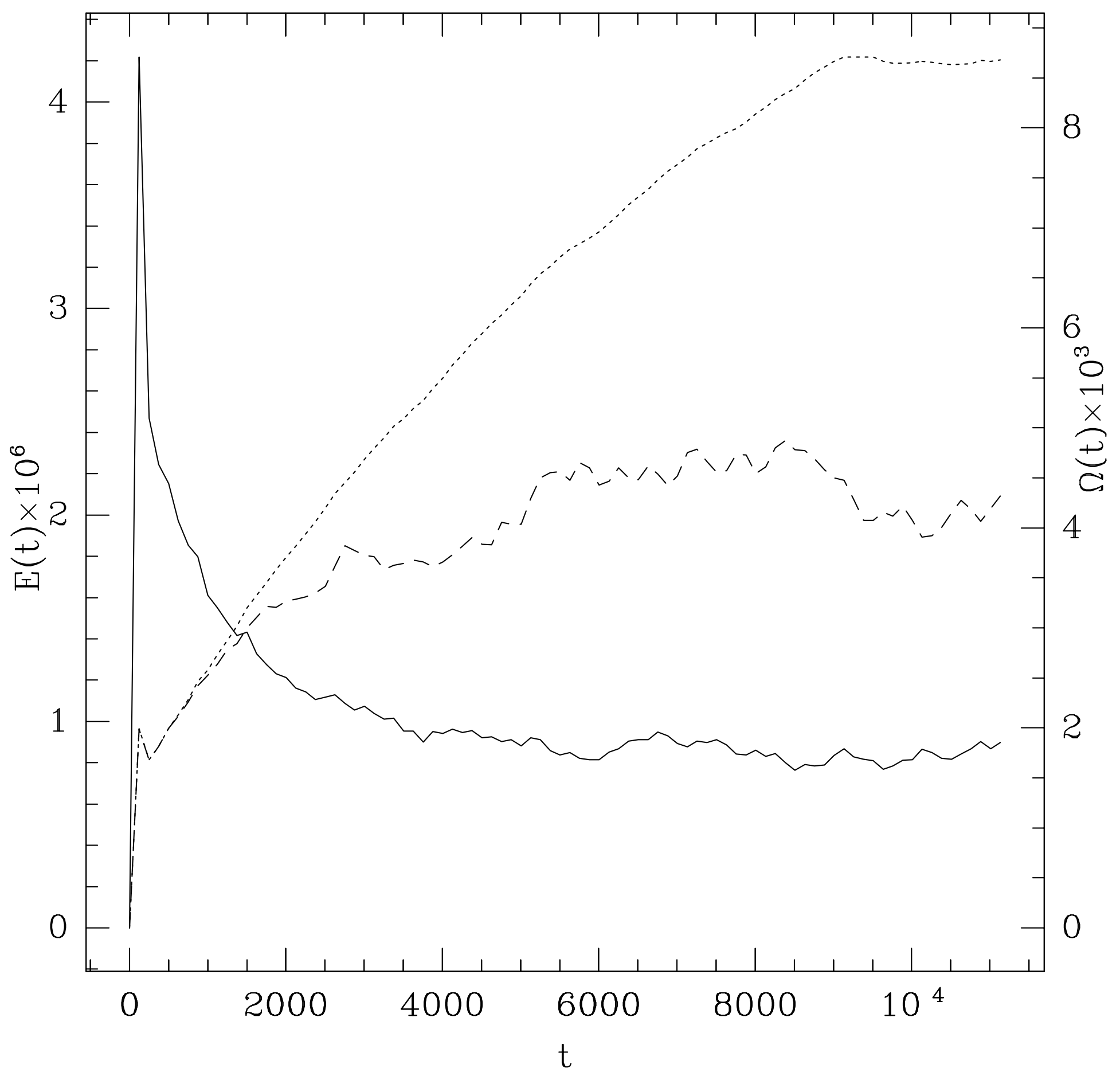




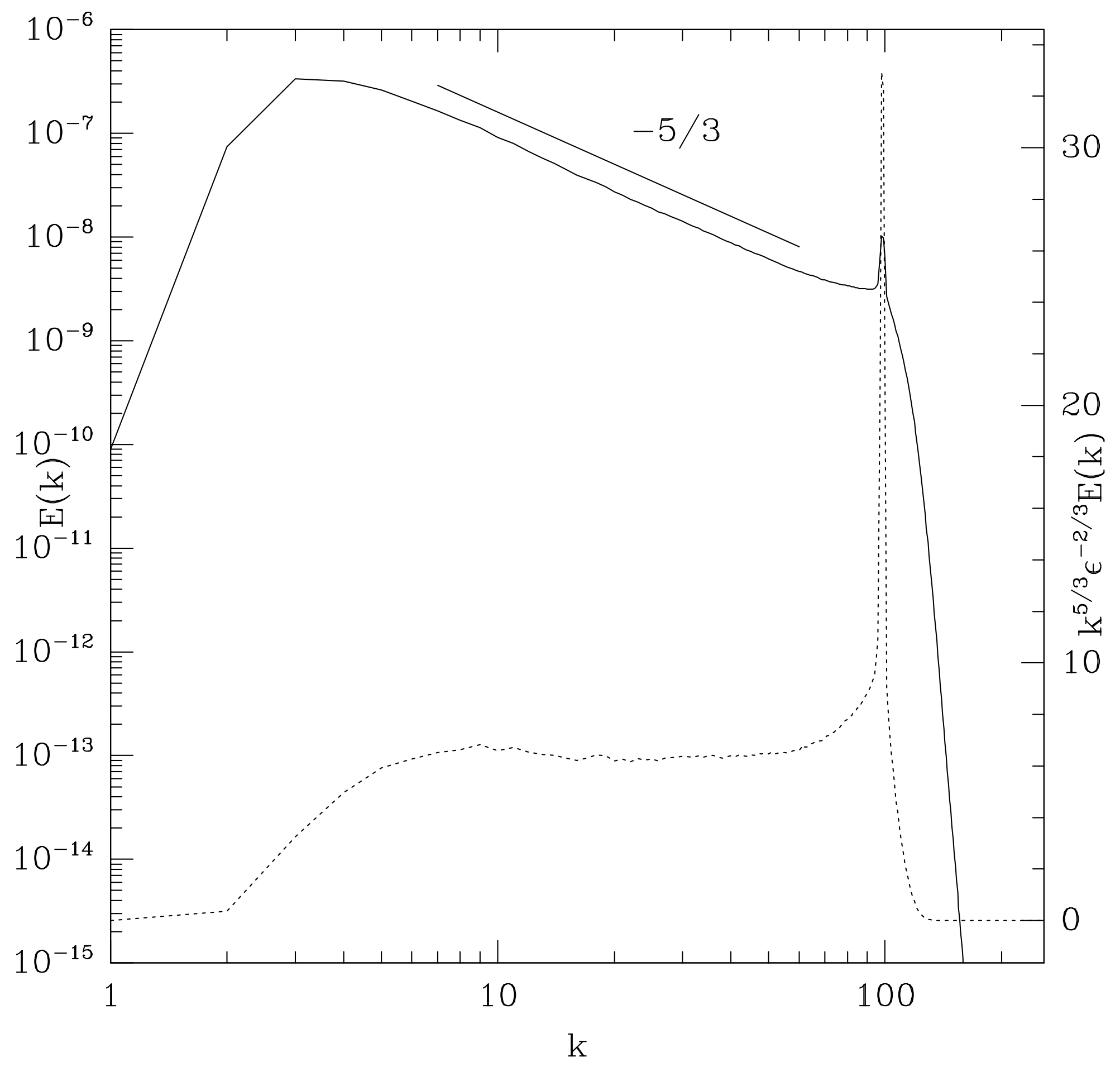




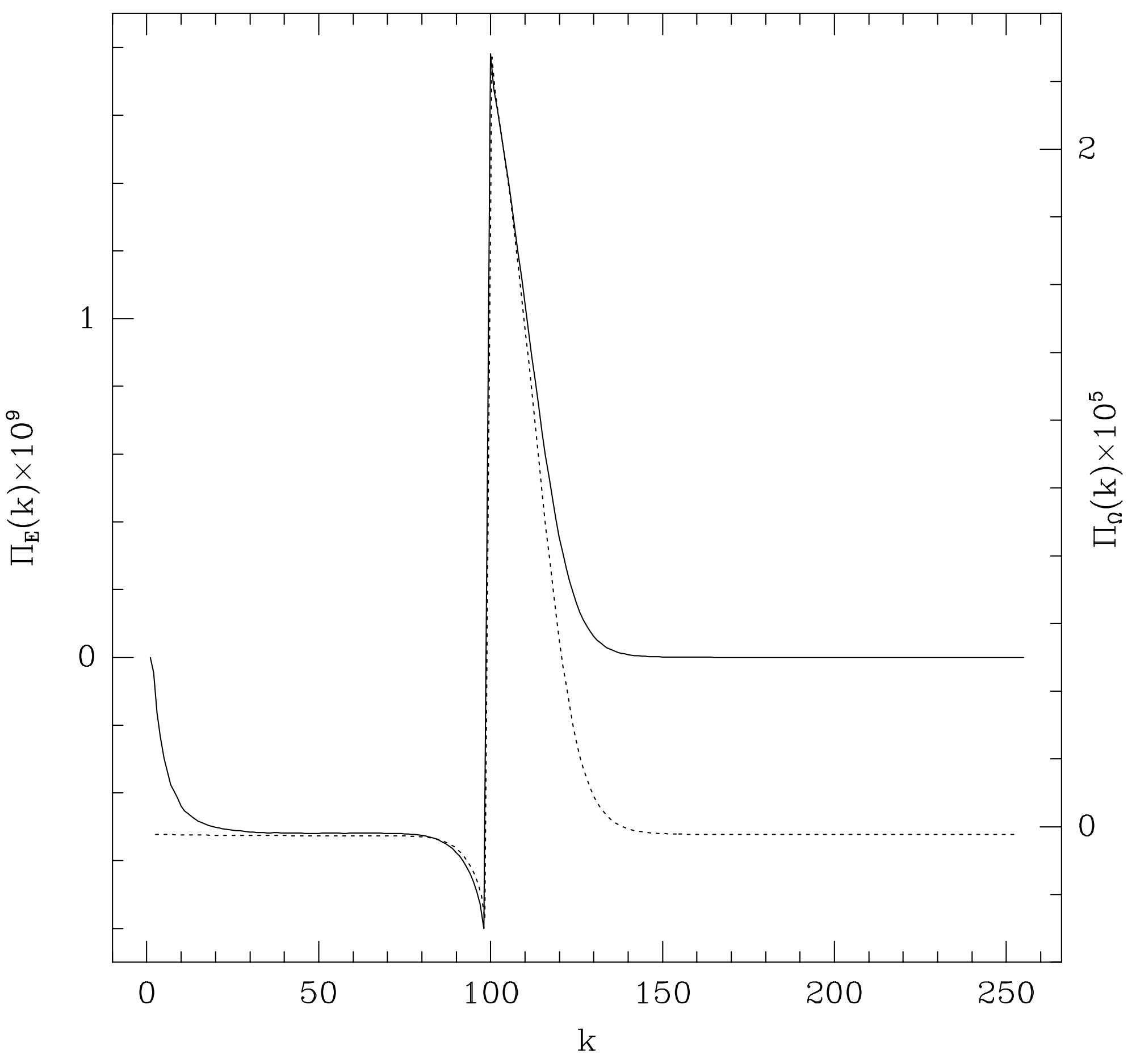




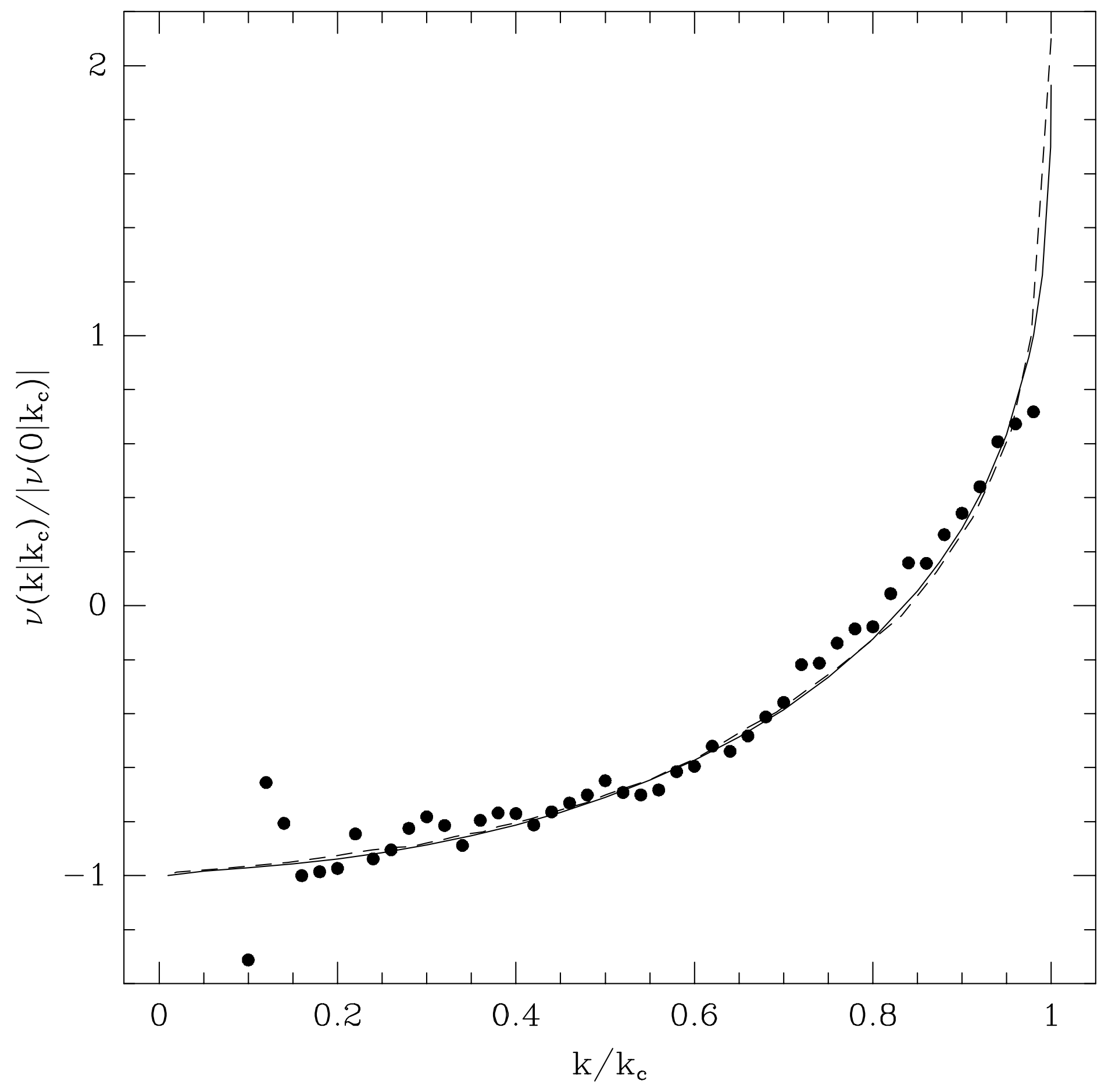




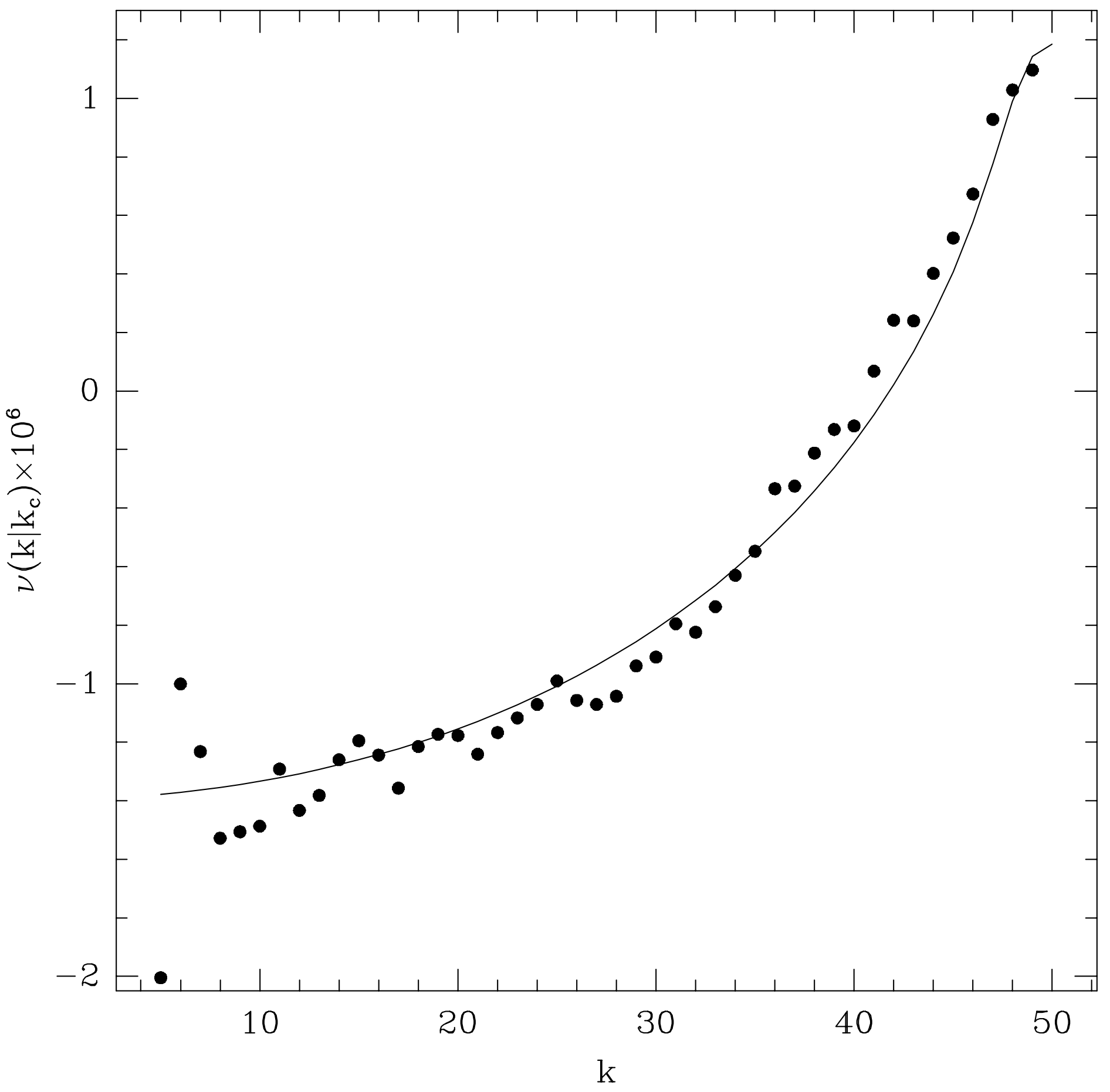

